\title{
La rebelión del Rif (1958-1959)
}

\author{
M. ${ }^{a}$ CONCEPCIÓN YBARRA*
}

\begin{abstract}
RESUMEN
La rebelión de las tribus rifeñas contra el gobierno de Marruecos, dos años después de la independencia de este país magrebi, es uno de los acontecimientos más ignorados de la historia del imperio alauíta. El famoso

Abdelkrim el Jattabi estuvo involucrado en dicho conflicto a la vez que el gobierno de Francia dirigido por De Gaulle. La misma España fue acusada de proteger a los rebeldes rifeños que amenazaban no sólo la estabilidad del nuevo régimen marroqui sino el mantenimiento del predominio francés en Argelia. En este trabajo se analizan las causas y consecuencias del «último levantamiento rifeño".
\end{abstract}

PALABRAS CLAVE Marruecos. Independencia. Relaciones internacionales. Conflictos rifeños. Abdelkrim. Implicación extranjera.

\section{ABSTRACT}

The rebelion of the riffian tribes against Morocco government, two years after the independence of this mogrebi country, is one of the most ignored events of the alaouit Empire. The famous Abdelkrim El Khatabi was involved in said conflict at the same time that the french goverment ruled by De Gaulle. Spain itself was accused of protecting the riffian rebels which threatened not only the stability of the new moroccan government but also the continuation of the french hegemony in Algeria. This report analizes the origin and consecuences of the "last riffian revolt".

\section{KEY WORDS}

Morocco. Independence. Foreign relations. Riffian conflicts. Repression. Abdelkrim. Foreign participation.

- UNED. 
La independencia de Marruecos (marzo-abril de 1956) no consiguió la sumisión de todo el Blad es Siba o territorio tradicionalmente disidente del poder temporal del Sultán. Tampoco se mejoraron las relaciones hispanomarroquíes debido a las continuas reclamaciones por parte del Gobierno marroquí de los territorios que aún España poseía en esa región. Este trabajo de investigación pretende aportar alguna luz sobre uno de los conflictos más desconocidos, aunque no menos problemáticos, en que se vio implicada España: la rebelión de las tribus rifeñas contra el Gobierno de Marruecos dos años después de haber sido declarada la independencia.

El Rif es uno de los territorios del nordeste de Marruecos, alrededor de Alhucemas, habitado por tribus bereberes de las que la más famosa es la de Beni Urriaguel. Genéricamente es la cadena montañosa de la zona norte de Marruecos ocupada desde la antigüedad por tribus bereberes de reconocido carácter rebelde y que fue parte integrante del Protectorado español. Esta región se pacificó en 1926 tras la rendición del histórico líder rifeño Abdelkrim el Jattabi. El autoproclamado Emir del Rif mantuvo en jaque a las tropas españolas y francesas durante varios años siendo el ejecutor del tristemente célebre desastre de Annualen 1921 y el artífice de la Républica del Rif.

La pacificación de la tribus rifeñas fue conseguida por el Gobierno de Primo de Rivera con la ayuda francesa tras la rendición a Francia del "Emir Abdelkrim". El Gobierno francés le recluyó en la Isla Reunión desde mayo de 1926 hasta 1947, año de su liberación, conseguida gracias a una hábil maniobra del Gobierno egipcio. Cuando Francia decidió trasladarle a la metrópoli, tras un confinamiento de 21 años en la lejana colonia francesa, al detenerse el barco en que viajaba en Port Said para realizar una escala técnica, Abdelkrim fué conducido al Cairo con toda su familia y enseres como protegido del Rey Faruk.

Desde 1927 y hasta el 20 de agosto de 1953, fecha del destronamiento y forzado exilio del sultán Mohamed ben Yusef, España no tuvo mayores problemas en toda la zona del Protectorado español ni con los marroquies ni con las autoridades francesas. Sin embargo, hasta el final del Protectorado los mandos militares mantuvieron una constante preocupación y vigilancia sobre las tribus rifeñas. Por la proximidad del Rif a los presidios de Ceuta y Melilla era necesaria la buena armonía entre protectores y protegidos.

A partir de agosto de 1953, momento en que Francia obligó a Mohamed $\mathrm{V}$ a abandonar el trono de Marruecos, las tribus rifeñas comenzaron a organizarse en contra del Gobierno francés con el beneplácito y la ayuda de la Alta Comisaría española. En 1955 los rebeldes rifeños habían 
creado un Ejército de Liberación para, mediante actos de fuerza, obligar al Gobierno francés a reinstaurar al sultán Ben Yusef y a conceder la independencia a Marruecos. Cuando en 1956 España transfirió los poderes administrativos al nuevo Estado marroquí, la zona rifeña quedó abandonada a su suerte o en manos de caídes procedentes del sur marroquí. Estos representantes del Sultán no poseían el suficiente prestigio y consideración para que todas las tribus rifeñas se les sometieran y cesasen en sus ataques contra las fuerzas gubernamentales marroquies y las tropas francesas que aún permanecian en Marruecos. Sin embargo, la veneración que el pueblo rifeño tenía por su Rey hizo que muchos militantes rebeldes, pertenecientes a los grupos armados del Ejército de Liberación, escucharan los consejos del Monarca y se afiliaran a las Fuerzas Reales marroquíes que se estaban creando bajo el patrocinio del príncipe Hassan. En julio de 1956, en una rápida visita de Mohamed $V$ a la zona rifeña, el Sultán había afirmado que la integración del Ejército de Liberación en la Fuerzas Reales era un gran paso en el camino de la estabilidad interna!

\section{ORIGEN DE LA DISIDENCIA}

Varios fueron los detonantes que originaron que la región rifeña se rebelara contra el Gobierno marroquí a finales de 1958, a los dos años de haber conseguido Marruecos su independencia.

En primer lugar, el rey Mohamed $V$ nunca tuvo en consideración a la zona rifeña, a pesar de haber sido uno de los territorios con más fuerza en el movimiento anti-francés para el regreso del Sultán y de que los rifeños hubieran sido los primeros voluntarios en la formación del "Ejército de Liberación». El Monarca no había olvidado los graves problemas que las tribus de las montañas del Rif, en su empeño de conseguir una república independiente, habían causado a su padre, el sultán Muley Yusef, hasta su muerte en 1927.

La vecina Argelia, todavía provincia metropolitana de Francia, se encontraba en guerra civil desde 1954. La mayoría de la población árabebereber era partidaria de la total independencia, contra el parecer del ejército francés y de la población gala radicados en la "provincia" africana, que abogaban por una "Argelia francesa». El tráfico de armas que se realizaba por la frontera del nordeste marroquí hacia el oeste argelino estaba implicando a muchos rifeños y la crisis político-militar argelina di-

Ver el diario «España», de Tánger. 16-VII-56. 
ficultaba el natural paso de trabajadores temporeros marroquies hacia los campos argelinos.

Por otra parte, las reformas económicas y políticas del nuevo Marruecos no contemplaban el Rif. Su precaria agricultura no recibió ayuda alguna en una época de sequía y de verdadera hambruna. Se enviaron caídes del sur para gobernar a los rifeños en vez de otorgar esa misión administradora a los propios líderes y jefes de tribus del norte marroquí. Las minas del Rif, único recurso de exportación de la zona norte, continuaron en manos extranjeras que las explotaban y esquilmaban. $Y$ como última humillación, ningún representante del Rif figuraba en los cuadros del Gobierno marroquí, debido quizás a la desconfianza del Rey y de los líderes nacionalistas del "Partido de la Independencia». El Istiqlal, partido mayoritario en el Gobierno marroquí y con gran influencia en las decisiones reales, era de mayoría árabe y consideraba al bereber Rif demasiado pro-español y por lo tanto traidor a la "causa democratizadora». Aunque, paradójicamente y con un alto oportunismo, el 30 de septiembre de 1956 el líder del Istiqlal, Allal el Fassi, había pronunciado un discurso en Rabat en el que negaba la instauración de la democracia en el Imperio jerifiano con estas palabras: "Nosotros no conocemos la democracia. Son los principios del Islam los que se deben aplicar en Marruecos" ${ }^{2}$. De esta manera quiso convencer a los rifeños de que su partido estaba supeditado a los mandatos de la religión musulmana y no a los dictados occidentales.

Finalmente, el histórico, anciano y añorado líder rifeño Abdelkrim, que en diciembre de 1947 había creado en El Cairo el Comité de Liberación del Norte de África, se negaba a volver a Marruecos con la excusa de que aún no se había conseguido la auténtica independencia. El verdadero motivo era su particular fobia hacia el Istiqlal ${ }^{3}$. Desde El Cairo continuaba «el Emir» enviando mensajes de apoyo a una rebelión rifeña contra un Gobierno, según él, corrupto y que tenía secuestrado al Sultán. El hijo mayor del "Jattabi", representante suyo en Marruecos, visitó al Rey en mayo de 1958 para expresarle los recelos de su padre contra El Istiqlal ${ }^{4}$, sobre todo contra su líder Allal el Fassi, con quien había roto relaciones en

Ver “España», de Tánger. 1-X-56.

Archivo Presidencia de Gobierno (APG). Legajo 34. Carpeta 11. Julio 1958. Manifiesto de Abdelkrim. Circular del Comité Árabe de Liberación Magrebi. Abdelkrim contra las afalsas independencias de Túnez y Marruecos", 24 de julio de 1958 . Todo este artículo en Ybarra, M. Concepción: Tesis Doctoral: La acción española en la descolonización del Magreb. Rivalidad hispano-francesa en Marruecos. (1951-1956). UNED, 1997.

4 APG. Leg. 33, carp. 10. "Enfrentamiento entre Abdelkrim y Ai Fassi». 29-5-1958. 
octubre de 1956 al constatar las ambiciones «oportunistas» del jefe istiqlalí. Las poblaciones rifeñas confiaban en que Abdelkrim iba a regresar para liberarles de la "dictadura" impuesta por el Gobierno de Rabat.

\section{PROGRESIÓN DEL PROBLEMA RIFEÑO}

El desarrollo de los acontecimientos conocidos como "Rebelión del Rif», según las noticias que llegaban a España desde Tetuán, fue el siguiente:

En mayo de 1958, poco después de haberse solucionado el conflicto hispano-marroquí originado por los ataques del "Ejército de Liberación" del sur marroquí contra las tropas españolas de Ifni y del Sáhara, Mohamed V, debido a la crisis en que se encontraba sumido su país, nombró un nuevo Gabinete ministerial con participación mayoritaria del partido del Istiqlal. Ello dió lugar a un mayor descontento entre las tribus rifeñas que clamaban por la vuelta de Abdelkrim. El Rey, tras destituir al Primer Ministro Si Bekkay, nombró Presidente del Gobierno y de nuevo Ministro de Asuntos Exteriores, a Ahmed Balafrej y al progresista Buabid, anterior Embajador en París y últimamente Ministro de Finanzas, como Vice-Presidente y Ministro de Economía y Agricultura. Balafrej había conseguido su objetivo: un Gobierno dominado por el Istiqlal. Ocho ministros pertenecian al partido y los otros dos eran independientes. El líder de la izquierda del Istiqlal, Mehdi ben Barka, continuaba presidiendo la Asamblea Consultiva desde otoño del 56. Sin embargo, a pesar del predominio de este partido en la vida marroquí, la crisis interna que estaba comenzando a padecer el Istiqlal se fue agudizando con los sucesos rifeños.

Cuando en agosto de 1958 aparecieron en las calles de Tetuán, antigua capital del ex-Protectorado español, varios letreros con vivas al "Generalísimo" Franco, se dio pie a la sospecha de una connivencia entre los rifeños y los españoles. Inmediatamente, varios marroquíes de la expolicía secreta fueron destituídos por su colaboración con las autoridades españolas. Estos antiguos guardianes del orden comentaron que en la zona del Rif existía una gran corriente contra el Gobierno de Marruecos y confirmaron que habían salido para Madrid cuatro marroquies amigos de España a fin de exponer a Franco la situación tan crítica que existía en dichos territorios ${ }^{5}$. Las promesas de regeneración económica y agrícola ex-

APG. Leg. 34. Carp. 12. Agosto de 1958. 
presadas por el Rey cuando visitó en febrero la zona norte, con motivo de emprenderse en la región la "operación arado" ", se habian visto frustradas al estar el Rif cada día más empobrecido y sometido a criterios ajenos, produciéndose el desencanto en su población. Al mismo tiempo fueron colocados en Tánger varios carteles subversivos contra el Rey y a favor de Nasser, principal líder nacionalista africano que había convertido Egipto en una República Árabe. La Prensa atribuyó su autoría al Partido Comunista marroqui ?

Uno de los líderes rifeños más considerados por Mohammed V, el Dr. Khatib, ex-Jefe y principal instigador del Ejército de Liberación Marroqui en 1955, fue recibido en Palacio para tratar con el Rey sobre la situación interna marroquí y la responsabilidad del Istiqlal. El Monarca le dió sólo buenas palabras, pero no actuó en consecuencia ${ }^{8}$. Sin embargo, se apresuró a nombrar al general Mizzian, antiguo militar procedente del Rif al servicio de España los últimos treinta años, Jefe de todas las Fuerzas Armadas Reales de la Zona Norte ${ }^{9}$. La población rifeña acogió este nombramiento con satisfacción debido al prestigio del general Mizzian. A pesar de ello, en las montañas se observaba nerviosismo y se esperaba un levantamiento de las cabilas del Rif ya que "habían perdido la fe en el Rey". Mizzian, temiendo problemas y levantamientos como los que ya estaban surgiendo en Nador y que tanto preocupaban a los melillenses por su proximidad a dicha Villa, había puesto a sus tropas en situación de máxima alerta y dispuso las medidas necesarias para proteger a los europeos a toda costa. Al fin, militarizó toda la zona norte de Marruecos ${ }^{10}$. Esta actitud fue muy bien vista por la población española que aún permanecía en la región. Algunas cabilas reclamaban más independencia y las tribus Beni Urriagel, Beni Esnassen y Tensaman comenzaron a sublevarse contra las autoridades reales. Al hacer aparición una bandera española en Tizzi Ifri, el Gobernador aprovechó para denunciar a los rebeldes rifeños como traidores a Marruecos y amigos de los españoles que anteriormente dominaban la región. El Ministro de Defensa y el general Mizzian tuvieron que acudir a entrevistarse con los cabecillas rifeños para poder poner paz en la zona. Las tribus exigían la desaparición del Istiglal antes de acceder a los requerimientos del jefe militar. Muchos marroquies del norte huian de sus

Archivo del Ministerio de Asuntos Exteriores (AMAE), Legajo Reservado 6.565/55 "Actividad política de Mohamed V". 19-2-1958.

APG. Leg. 34. Carp.12. Agosto de 1958.

APG. Leg. 34. Carp.12. Entrevista del Dr. Abdelkrim Al Jatib. 9 de agosto de 1958.

APG. Leg. 34. Carp.13. Situación militar en Marruecos. 2 de septiembre de 1958

APG. Leg. 34. Carp. 15. 22-X1-1958. 
pueblos y aldeas y se refugiaban en Ceuta o Melilla esperando el apoyo de España ".

No sólo existía una gran frustración entre los rifeños y españoles residentes en Marruecos, por la política llevada a cabo por el Gobierno istiqlalí, sino que otras personalidades notables del régimen marroquí estaban, por esas fechas, manifestándose contra las disposiciones gubernamentales. Tal era el caso del antiguo líder del nacionalismo tetuaní y anterior Embajador de Marruecos en Madrid, Abdelkhalek Torres, quien como consecuencia de su violento discurso radiofónico desde Tetuán, en el que se habia permitido criticar al Gobierno considerándole culpable del caos económico, del paro y de la miseria del antiguo Protectorado español, había salido precipitadamente de Marruecos rumbo a su Embajada del Cairo ${ }^{12}$. Por otra parte, varios marroquíes residentes en Melilla eran señalados como posibles contrabandistas de armas y como participantes en una red de espionaje en la que también se implicaba a algunos españoles de los Presidios. La situación del Rif y sus alrededores se iba volviendo peligrosa, incluso se acusaba a los franceses de ofrecer armamento y dinero por la zona norte para fomentar una rebelión en la que también se viera implicada España ${ }^{13}$.

El Gobierno trancés se mantenía bien informado sobre la situación del Rif gracias a los continuos comunicados enviados por su Embajador en Madrid. El embajador La Tournelle opinaba que las Plazas españolas corrian también peligro de ser atacadas por su proximidad al escenario de los enfrentamientos armados. Entonces, el Ministerio de Exteriores francés se vió en la necesidad de alertar al Gobierno marroquí de la "extrema gravedad» que las agresiones a los territorios españoles comportaría para las relaciones hispano-marroquies. "Marruecos sería el responsable de atentar contra la paz y soberania de territorios españoles" ${ }^{14}$. Aunque el Quai d'Orsay era consciente de que los rifeños estaban en contra del colonialismo francés y participaban en la rebelión argelina suministrando armas y hombres al Frente de Liberación Nacional (FLN) de Argelia. Por otra parte, y fiel a su tradición, el Foreign Office británico también estaba siguiendo con preocupación los sucesos del Rif. Su punto de vista coincidía con el francés pero era aún más crítico con España a la que

Ibid. APG. Leg.34/15. 26-X1-58

APG. Leg.34. Carp.13. IX-1958. Torres era desde 1957 Embajador en Egipto.

APG. Leg. 34. Carp. 13. "Situación alarmante en Marruecos". 24-IX-58.

${ }_{14}$ Ministère des Affaires Etrangers (MAE). Serie Europa. 44-60-Espagne. Vol. 248. 1958-

1960. Dependence d'Outre Mer-Maroc. 26-IX-1958. 
consideraba culpable del levantamiento de la antigua zona española de Marruecos ${ }^{15}$.

Mientras, las minas del Rif seguían proporcionando a la industria española un millón de Tm. de hierro al año según un informe oficial. Para evitar esta explotación colonizadora el Gobierno marroquí se propuso cercar económicamente a Ceuta y Melilla desviando carreteras, dificultando la traída de aguas y endureciendo las medidas aduaneras. Incluso, desde algunos sectores, responsabilizaban al Rey de fomentar el nacionalismo de los líderes rifeños: el Dr. Khatib y el Capitán Ahardán, antiguo Gobernador de Rabat, habian creado en octubre de 1957 el Movimiento Popular con el consentimiento del Monarca "no sólo para que fuera una fuerte oposición al excesivo poder del Istiqlal, sino también, para que se creara una situación de inestabilidad en la zona norte y poder así tener mayor fuerza ante la ONU en la reclamación de las Plazas españolas en Marruecos" "16. Mohamed $V$ intentó atraerse a los rifeños con la devolución oficial a la familia de Abdelkrim el Jattabi de las tierras requisadas por los españoles ${ }^{17} y$ decidió pagar mensualidades a los hijos del «Emir del Rif» con el fin de que toda la familia Abdelkrim volviera a Marruecos ${ }^{18}$.

En octubre de 1958 la situación en la zona norte se fue complicando por momentos. Continuos actos de sabotaje pretendían llamar la atención de la población. El Gobierno marroquí, para debilitar a los rebeldes, decidió detener a los líderes rifeños, Khatib y Ahardán, acusándoles de ser "los instigadores de manifestaciones antigubernamentales donde se pronunciaban discursos incitando a la revuelta y la agitación" ${ }^{19}$. El Consejo de Ministros, reunido con el Rey en Fez el 3 de octubre, ordenó una investigación sobre los líderes rifeños detenidos. Según el diario pro-istiqlali Al Alam "los sucesos en el norte estaban convirtiéndose en una anarquía a la que se debía poner coto". Este periódico acusaba a "potencias extranjeras" de surtir de armas a los grupos rebeldes del Atlas Medio y del Rif que luchaban contra el Gobierno por ser éste de tendencia istiqlalí ${ }^{20}$.

${ }^{15}$ Public Record Office (PRO) Foreign Office (FO), 371. n. 9138 445: Relaciones hispanomarroquies, 1958-59

16 APG. SMS Leg. 36. Carp. 17. El Periódico El Sahafa de El Cairo denunciaba la "connivencia" entre el Rey Mohamed $V$ y el Movimiento Popular riteño para restar fuerza al Partido del Istiqlal. 5-11-1959.

Ver Moumen Diouri, "La realidad de Marruecos", pág 124. Madrid, 1988.

AMAE. R.7.266/6: Mohamed Ben Abdelkrim el Jattabi. Sus Memorias.

APG. Leg. 34. Carp. 17. Información del Rif. 2-X-1958.

2 APG. Leg 35. Carp. 12. Octubre de 1958 
La prensa internacional también comenzó a interesarse por el movimiento de oposición, surgido en la región rifeña, contra el Gobierno de Balafrej ${ }^{21}$. Asimismo, resaltaba los problemas internos de Marruecos y la voluntad del Rey y su Gobierno por cortar cualquier disidencia. Una de las actuaciones más conflictivas, por sus implicaciones con el exterior y sobre todo con la URSS ${ }^{22}$, en un momento en que Marruecos estaba estableciendo buenas relaciones con los países del bloque comunista, fue la detención de Alí Yata, Secretario General del Partido Comunista marroquí, que trataba de potenciar su partido con la ayuda de la Internacional Comunista. Le Figaro se preguntaba si se habia emprendido en Marruecos la lucha contra el PC. La prensa española, debido a la censura impuesta por el régimen, apenas comentaba el encarcelamiento del líder comunista ni las noticias del levantamiento rifeño. En noviembre de 1958 y ante el recrudecimiento del conflicto, algunos periódicos como el Ya, Madrid, ABC, etc... incluyeron breves referencias a los sucesos. Sólo la entrevista de Mohammed $\mathrm{V}$ y Balafrej con una delegación rifeña recibió mayor atención ${ }^{2}$. Varios periódicos explicaban que los cincuenta delegados rifeños habían expuesto al Monarca que la causa de la "agitación rifeña» era la grave situación social y económica en que se encontraba la zona norte. El 15 de noviembre el Rey visitó esta conflictiva región como muestra de su sincera preocupación. La prensa resaltaba ${ }^{24}$ el entusiasmado recibimiento con que fue acogido el Sultán en Tetuán y Tánger. Por aquellos días se conmemoraban en todo Marruecos las Tres Jornadas Gloriosas (la XXXI exaltación al Trono del sultán Mohamed ben Yusef, el tercer aniversario del retorno de Mohamed $V$ y la Independencia del pais). Al frente del desfile militar, celebrado el día 15 en Tetuán, figuraban los generales más prestigiosos y formados en los dos paises "protectores": Ben Mizzian y Ben Kittani. El Gobierno de Madrid intentaba convencer a la opinión pública española de que esta visita tan solemne del Rey a la zona norte, aún de influencia española, significaba un acercamiento hispano-marroquí.

Pese a todos los esfuerzos del Monarca por apaciguar a las poblaciones rifeñas y el envío de la Comisión Real ${ }^{\approx 5}$ para entablar conversaciones con

21 Le Figaro, 3-10-1958, denuncia a los marroquies del Rif que están atentando contra el Gobierno haciéndose fuertes en algunos poblados. Aunque destaca que los rifeños se consideran fieles a Mohamed $V$ pero no a sus ministros.

2 APG. SMS. Leg.24. Carp.16. En diciembre se estableció oficialmente en Marruecos la Embajada de la URSS.

23 Ya. 12-11-1958.

24 Madrid. 17-X1-1958

a El diario Madrid publicó el 13 de diciembre unas delaraciones del miembro de la Comisión Si Abderrahman Anegai en las que expresaba su solidaridad con las reivindicaciones rifeñas, afirmando que "las Tribus eran totalmente leales al Rey" aunque estuvieran en contra del Gobierno. 
los jefes de tribus y conocer sus necesidades "in situ", los atentados ${ }^{26}$ se sucedian y el Gobierno de Balafrej se tambaleaba. Al fin, el 28 de noviembre, estalló la crisis política. El vicepresidente Buabid ${ }^{27}$ presentó su dimisión al Rey con gran contrariedad de Balafrej. La reciente elevación de impuestos a los agricultores rifeños había causado la protesta y descontento entre las tribus y el rechazo de toda la zona norte contra un Gobierno "que así les conducía a la miseria». El Ministro de Economía no encontró mejor salida a tanto conflicto que abandonar su puesto. El Rey también temía que su Monarquía se viera afectada en esa crisis política y social, sobre todo por la influencia antimonárquica de la revolución de Iraq con la muerte del joven Rey Faisal, según expresaba la prensa española. Mohamed $V$ se apresuró a publicar la Carta Real, anticipo de la prometida Constitución democrática, y volvió a enviar al general Mizzian a parlamentar con los jefes tribales, que en estos días habían reunido una "guerrilla" de más de 25.000 hombres en plan de "marcha" hacia Nador. Los informes oficiales que las autoridades militares españolas en Marruecos enviaban al Ministro subsecretario de la Presidencia del Gobierno, almirante Carrero Blanco, decían que "cuando llegaron a Monte Arruit, Mizzian les habló y convenció para que no entraran en esa Villa, vecina de Melilla, que se encontraba atemorizada y paralizada» ${ }^{26}$. Se liberó a los líderes rifeños, Dr. Khatib y Capitán Ahardán, quienes se apresuraron a celebrar una conferencia de prensa donde denunciaron que mientras ellos permanecían 158 días en prisión, las autoridades habían intentado eliminar el Movimiento Popular que ellos presidían. Exhortaban a su pueblo a permanecer al lado del Rey y pedían a éste que dijera la verdad al país sobre la situación real de Marruecos y el fracaso del Gobierno «istiqlalí» 29 . Aunque muchos rebeldes estaban detenidos, a mediados de diciembre el Ejército de Liberación del Rif logró tomar todos los accesos a Villa Sanjurjo (Alhucemas), permitiendo el paso solamente a los españoles. Sus exigencias para deponer las armas eran: la salida de los caídes extraños a su región y el nombramiento de sus líderes como principales autoridades de la zona, a ejemplo de lo que anteriormente habian hecho los españoles

26 Uno de los más graves fue el que se produjo el 24 de noviembre en Jemsset, a $80 \mathrm{~km}$ de Rabat. Una bomba de gran potencia estalló entre la multitud que participaba en las Tres Jornadas Gloriosas produciendo varios muertos y más de cien heridos. Ver Madrid, 25-XI-58 y APG. SMS Leg.34. Carp. 12. El Tribunal de Justicia de Rabat condenó a muerte a uno de los "cabecillas" rifeños, Dris el Rifi, tras un juicio cuyo interrogatorio se basó en averiguar el grado de complicidad que España tenía en las revueltas rifeñas.

27 APG. Leg. 34. Carp.16. Una "Nota Informativa» dirigida a Carrero Blanco acusaba a Buabid de haber comprado armamento en Yugoes!avia destinado al ELM.

æ APG. SMS. Leg.34. Carp. 16. «Nota Informativa» literal del 5-XII-58.

a lbid. "Situación en el Rif". 4-XI-1958. 
cuando gobernaban en el Rif. También los estudiantes rifeños se pusieron en huelga ante la declaración de hacer oficial el bachillerato francés y la supresión del español. Asimismo, protestaban por las dificultades de las autoridades marroquíes para reconocer los títulos de las Universidades españolas y no así de las francesas ${ }^{x}$.

Los dirigentes del Istiqlal no se ponian de acuerdo para solucionar la crisis del Rif y el Rey tuvo que intervenir forzando la dimisión de Balafrej y negándose a las proposiciones del ala izquierdista de dicho partido. El 17 de diciembre y tras arduas discusiones, el Monarca encargó a Abdallah Ibrahim, antiguo dirigente del Istiqlal, que formara un Gobierno de representación nacional ${ }^{31}$. Por fin y tras el rechazo del Rey a varias listas de ministros presentadas por Ibrahim, el 24 de diciembre se constituyó el nuevo Gobierno con la aprobación real. Debido a que muchos de los nuevos ministros pertenecian al ala izquierdista del Istiqlal, los seguidores de Allal el Fassi, de tendencia moderada, manifestaron su oposición al nuevo Gobierno. Tampoco los rifeños estuvieron satisfechos con la decisión real, ya que ninguno de sus representantes figuraba en el nuevo Gabinete. Mientras tanto, en Francia era elegido Presidente de la $V$ República el General De Gaulle, quien desde el "Golpe" del 13 de mayo de 1958 había desempeñado el cargo de Primer Ministro del Gobierno francés. Se confiaba que De Gaulle solucionaría rápidamente la crisis de Argelia.

En enero de 1959, el Rey decidió acabar con las protestas rifeñas de una forma drástica: grandes contingentes de tropas del Ejército Real, al mando del príncipe Hassan, fueron desembarcadas en Tánger y Alhucemas, ante la dificultad de alcanzar el Rif por vía terrestre ${ }^{2}$. El heredero del Trono, acompañado del coronel Ufkir, estableció su cuartel general en Fez con el fin de dirigir mejor las operaciones contra los rebeldes rifeños y del Atlas Medio, según un comunicado oficial del Gobierno. La Aviación Real, asesorada y dirigida por técnicos y pilotos franceses se encontraba dispuesta a actuar en la zona. Al sur del Rif, en las montañas del Atlas, las tribus bereberes habían comenzado también a levantarse contra el Gobierno. El caid Lahcen el Yussi, poderoso jefe bereber y antiguo Ministro de Interior en el primer Gabinete tras la independencia y exConsejero del Rey, se acababa de sublevar refugiándose en las montañas de Skura Auit Serruchen, a $70 \mathrm{kms}$. al sur de Fez. Estaba reclamado por haber participado en la conspiración antigubernamental urdida por el jefe

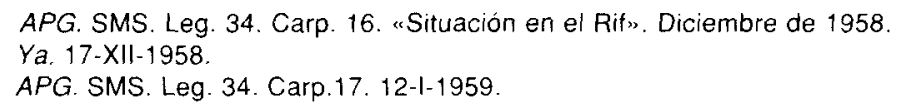


bereber y ex-Gobernador de Tafilalet Addi $U$. Bihi a quien ya se estaba juzgando en el Tribunal de Rabat ${ }^{33}$. Se les acusaba a ambos de haber tratado con Francia el derrocamiento del Gobierno con el fin de que volviera el régimen colonial anterior, más favorable a los bereberes que el actual ${ }^{34}$. Mientras las Fuerzas del Ejército Real salían de Fez para combatir a los guerrilleros de El Yussi, Mohamed V reunía al Alto Comité de Defensa para redactar el Ultimatum a los rebeldes ${ }^{35}$. Este edicto real fué trasmitido en lengua árabe, chelja y otros dialectos bereberes a través de la radio, la prensa y las octavillas que lanzaron aviones de combate en las aldeas de las montañas y en las poblaciones de los valles. En él se comunicaba a todos los marroquíes que «el Rey ordenaba a los amotinados cabileños de las montañas que acataran la ley, volviendo el orden a sus regiones en un plazo de 48 horas", les advertía que en caso contrario sufrirían "cruel castigo". Al acabar el plazo, muchos cabileños volvieron a sus hogares procedentes de los refugios rebeldes de las montañas por miedo a perder no sólo la vida sino también por posibles represalias a sus familias. Sin embargo, en la propia región rifeña, alrededor de Alhucemas, los bereberes no sólo no se rindieron sino que prosiguieron sus escaramuzas contra la Fuerzas Reales, por lo que, el príncipe Hassan, pidió ayuda a Francia e hizo desembarcar un gran contingente de paracaidistas marroquies y franceses en las costas de Alhucemas. Había comenzado la lucha fratricida. EI Jefe de las Fuerzas Armadas "no iba a consentir" que unos pocos levantiscos bereberes pusieran en peligro la autoridad de su padre el Rey.

La prensa istiqlalí denunciaba a los "cabecillas» rifeños de estar apoyados por España y exigía la "urgente evacuación de las tropas españolas que todavía se encontraban en el antiguo Protectorado" ${ }^{3}$. Los medios de comunicación franceses también se preguntaban si detrás de los rebeldes rifeños se encontraba el Gobierno de Franco ya que varios españoles habían sido hechos prisioneros junto a los sublevados. Denunciaban la acogida que en Ceuta y Melilla se dispensaba a los rebeldes rifeños que huían de la represión real. Para reforzar sus argumentos incluían las declaraciones del príncipe Hassan sobre «las intrigas extranjeras» que estaban desestabilizando el país ${ }^{37}$. El 17 de enero el Gobierno marroquí envió

${ }^{33}$ El 1. de febrero Addi $\cup$ Bihi fue condenado a muerte con otros siete dirigentes bereberes. VerMadrid, 2-11-1959.

34 Madrid. 6-1-1959

36 APG. SMS. Leg. 34. Carp. 17 «Situación política en Marruecos». 12-1-1959.

* "Al/stiqlal» junto con el diario "Al Alam" no cesaban de acusar a España como instigado ra de la "rebelión del Rif".

APG. SMS. Leg. 36. Carp. 15. Enero de 1959. 
una protesta formal al Gobierno español, a través de su Embajador en Rabat, asegurando que entre los detenidos rifeños se encontraban algunos elementos españoles. Se había descubierto que el contingente armamentístico capturado a los sublevados era de procedencia española y causaban sorpresa y consternación las facilidades que las autoridades de Ceuta y Melilla concedían a los disidentes perseguidos ${ }^{3}$. Los líderes rifeños ya no pudieron seguir manifestando su adhesión al Monarca porque éste les había enviado a su hijo para que reprimiera la rebelión. Pese a la abrumadora superioridad armamentística del Ejército Real contra las tribus que se habian hecho fuertes en las montañas, los rifeños no se rindieron. Estaban dispuestos a defender sus derechos hasta que desapareciera el último guerrillero o el Rey atendiera sus reclamaciones.

Mientras tanto, la prensa francesa alertaba a la opinión pública sobre el peligro de una "guerra civil» en Marruecos a causa de la "sangre que se estaba vertiendo en el Rif $"{ }^{30}$. Sin embargo, los periódicos marroquíes de tendencia istiqlalí preferian resaltar la entrevista que el Ministro español de Asuntos Exteriores, Castiella, estaba manteniendo con Nasser en El Cairo. Aseguraban que la intención de España de seguir buscando la amistad árabe era recabar su apoyo para permanecer en sus colonias africanas ${ }^{40}$. La prensa y la radio egipcias, a su vez, atacaban al Rey de Marruecos, al Príncipe heredero y a las Fuerzas Armadas Reales por estar llevando a cabo un castigo excesivo en el Rif. Comentaban que "aunque los aviones franceses participaban en la represión contra las cabilas revueltas y pese a la tentativa del Rey y los reaccionarios, la revolución de Marruecos continuaba» ${ }^{41}$.

El 6 de febrero de 1959 Abdelkrim el Jattabi como Presidente del Comité Árabe pro Libertad del Magreb dirigió desde El Cairo un Manifiesto al pueblo marroquí combatiente en el que denunciaba al Rey y al Gobierno de Marruecos por haber enviado "aviones franceses mortiferos e incendiarios a aplastar $y$ aniquilar a los rebeldes del Rif». También acusaba al Monarca de elaborar "un doble plan satánico": ponerse de parte del «traidor Allal el Fassi" y "sumir al país en la anarquía y el desorden para favorecer el que Francia y sus agentes se apoderen de la patria". Según Abdelkrim si la revolución bereber triunfara «barrería a Francia de

Paris-Presse I'Intransigeant. París. 19-I-1959.

Le Figaro. 26-1-1959.

Al Istiqlal y Al Alam. 24-1-1959.

AMAE. Leg.R.7.266. Exp. 6. 5-II-1959. Y APG. SMS. Leg. 36. Carp. 17. "Al Sahafa». 5-II1959 
Marruecos y después barreria al Monarca y a sus partidarios agentes de Francia". Terminaba su comunicado exhortando a su pueblo a continuar con la «revolución» para conseguir no sólo la libertad e independencia del Rif sino "la victoria del nacionalismo árabe dotado de personalidad independiente, apartada del Oriente y del Occidente" ${ }^{42}$. Moumen Diouri, líder nacionalista marroquí y actualmente exiliado político, ha escrito que:

\begin{abstract}
"...mientras el 5 de enero de 1959 el Rey hace una llamada a los rebeldes insurrectos y les pide que depongan las armas, el Príncipe Muley Hassan instala su Estado Mayor en Tetuán y su ayudante el Coronel Ufkir establece los Tribunales que juzgarían y condenarian a los rebeldes. En la costa mediterránea desembarcaron veinte mil hombres (infanteria, caballeria, blindados, artilleria). Estas Fuerzas Armadas Reales, distribuidas en tres columnas, barrieron todo a su paso. Refugiados en las montañas, los rifeños fueron rodeados. Los combates tuvieron una violencia extrema, llegando la aviación real a bombardear las aldeas en febrero de 1959. La represión no perdonó a nadie, ni a las mujeres ni a los niños. Se contabilizaron ocho mil muertos y miles de heridos..." ${ }^{43}$.
\end{abstract}

Al tiempo que se agudizaban los problemas en las montañas del norte de Marruecos el poderoso partido del Istiqlal comenzó a escindirse en dos grupos; los moderados patrocinados por Al Fassi y Balafrej y los progresistas liderados por Ben Barka, Ibrahim y Buabid. Estos últimos contaban con el apoyo de la importante organización sindical Unión Marroqui del Trabajo (UMT) que amenazaba con una huelga general si no se atendian sus reivindicaciones. La facción izquierdista, finalmente, se organizó en un nuevo partido: la Unión Nacional de las Fuerzas Populares (UNFP). El Istiqlal, durante algún tiempo se dedicó a desprestigiar al nuevo partido y a sus líderes tachándoles de "comunistas". La crisis del Rif había conseguido desestabilizar al partido más importante de Marruecos. Mohamed $V$ permitió que ambos bandos del Istiqlal se disputaran el espacio político. Cuantos más partidos hubiera mayor poder de maniobra tendría el Monarca. Gracias a los continuos enfrentamientos entre los líderes del histórico Istiqlal, el Rey tuvo la ocasión, unos meses más tarde, de cesar al Gabinete Ibrahim y asumir personalmente la dirección del Gobierno ${ }^{44}$. La "disidencia» del Rif había sido aniquilada y El Jattabi tuvo que pedir el Amán del Rey en mayo de $1959^{45}$. Los pueblos Amazighs

42 APG. SMS. Leg. 34. Carp. 19. Traducción del Manifiesto del Comité Arabe pro Libertad del Magreb. 6-11-1959.

43 Ver Diouri: "La realidad de Marruecos", págs. 124-150. (Ob. cit.).

44 Ver Jean Ganiage: Histoire Contemporaine du Maghreb. Paris. 1994

45 AMAE. Leg. R. $7266 / 6$. Abdelkrim el Jattabi. Sus Memorias. 
(bereberes) ya no volverían a "levantarse" en Marruecos contra la autoridad gubernamental.

Una vez que la situación del Rif se había resuelto para el Gobierno marroquí, aunque no se hubiera cumplido el deseo bereber de igualarse social y políticamente con los demás ciudadanos árabes, el Monarca se dispuso a visitar los territorios rifeños para conseguir la sumisión de las tribus Beni Urriagel. Pacificada la zona, España, Francia y Marruecos dieron por terminada "La Rebelión del Rif». Sin embargo, Mohamed $V$ poco pudo disfrutar de su máximo prestigio y poder ejecutivo ya que moriría en febrero de 1961 a causa de una pequeña intervención quirúrgica en su tabique nasal. El Rey de Marruecos, de 51 años, héroe de la independencia y personaje mitificado por su pueblo, dió paso a una nueva etapa de la Historia de Marruecos protagonizada por su hijo primogénito y heredero del Imperio alauita, el Rey Hassan II. 